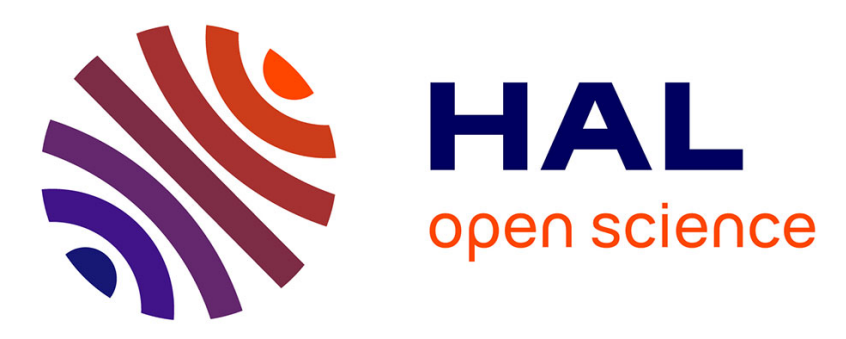

\title{
Periodic Oscillations in Leukopoiesis Models with Two Delays
}

\author{
Mostafa Adimy, Fabien Crauste, Shigui Ruan
}

\section{To cite this version:}

Mostafa Adimy, Fabien Crauste, Shigui Ruan. Periodic Oscillations in Leukopoiesis Models with Two

Delays. Journal of Theoretical Biology, 2006, 242, pp.288-299. 10.1016/j.jtbi.2006.02.020 . hal00258393

\section{HAL Id: hal-00258393 \\ https://hal.science/hal-00258393}

Submitted on 22 Feb 2008

HAL is a multi-disciplinary open access archive for the deposit and dissemination of scientific research documents, whether they are published or not. The documents may come from teaching and research institutions in France or abroad, or from public or private research centers.
L'archive ouverte pluridisciplinaire HAL, est destinée au dépôt et à la diffusion de documents scientifiques de niveau recherche, publiés ou non, émanant des établissements d'enseignement et de recherche français ou étrangers, des laboratoires publics ou privés. 


\title{
Periodic Oscillations in Leukopoiesis Models with Two Delays*
}

\author{
Mostafa Adimy ${ }^{a}$, Fabien Crauste $^{a}$, Shigui Ruan ${ }^{b}$
}

Year 2006

${ }^{a}$ Laboratoire de Mathématiques Appliquées, UMR 5142,

Université de Pau et des Pays de l'Adour,

Avenue de l'université, 64000 Pau, France.

ANUBIS project, INRIA-Futurs

Emails: mostafa.adimy@univ-pau.fr (M. Adimy),fabien.crauste@univ-pau.fr (F.Crauste)

${ }^{b}$ Department of Mathematics, University of Miami,

Coral Gables, FL 33124-4250, USA.

Email:ruan@math.miami.edu

\begin{abstract}
The term leukopoiesis describes processes leading to the production and regulation of white blood cells. It is based on stem cells differentiation and may exhibit abnormalities resulting in severe diseases, such as cyclical neutropenia and leukemias. We consider a nonlinear system of two equations, describing the evolution of a stem cell population and the resulting white blood cell population. Two delays appear in this model to describe the cell cycle duration of the stem cell population and the time required to produce white blood cells. We establish sufficient conditions for the asymptotic stability of the unique nontrivial positive steady state of the model by analyzing roots of a second degree exponential polynomial characteristic equation with delay-dependent coefficients. We also prove the existence of a Hopf bifurcation which leads to periodic solutions. Numerical simulations of the model with parameter values reported in the literature demonstrate that periodic oscillations (with short and long periods) agree with observations of cyclical neutropenia in patients.
\end{abstract}

Keywords: Leukopoiesis, hematopoietic stem cells, delay differential equations, stability, Hopf bifurcation.

\section{Introduction}

The process that leads to the production and regulation of blood cells is called hematopoiesis. It consists of mechanisms triggering differentiation and maturation of hematopoietic stem

\footnotetext{
*To appear in Journal of Theoretical Biology
} 
cells. Located in the bone marrow, hematopoietic stem cells are undifferentiated cells, unobservable directly (even though they can be tracked by markers), with unique capacities of differentiation (the ability to produce cells committed to one of blood cell types) and self-renewal (the ability to produce an identical cell with the same properties). Under the action of growth factors (molecules acting like hormones playing an activator/inhibitor role), hematopoietic stem cells produce differentiated cells throughout cell divisions until blood cells (white cells, red blood cells, and platelets) are formed and ready to enter the bloodstream.

We are interested here in leukopoiesis (see Fig. 1), the process by which white blood cells (also known as leukocytes) are produced, which is a sub-process of hematopoiesis. Like other blood cells, white cells are originated from a pool of hematopoietic stem cells. Under the action of mainly G-CSF (Granulocyte Colony Stimulating Factor), a growth factor only acting on the leukocyte line, hematopoietic stem cells differentiate in progenitors (the so-called CFU, Colony Forming Units), which in turn will produce precursor cells after a consequent number of divisions. After a few divisions late, leukocytes are formed and leave the bone marrow to enter the bloodstream.

Due to the number of divisions and the quantity of cells involved in leukopoiesis (or in hematopoiesis in general), issues may arise at different cellular levels and sometimes result in diseases affecting white cells. Among a wide variety of diseases affecting leukocytes, cyclical neutropenia is of great interest. It is characterized by a periodic decrease in the circulating neutrophil (white cells) numbers, from normal to low values, sometimes barely detectable [21]. Usually periods observed vary between 19 and 21 days, but longer periods up to 46 days have been reported in some patients [22]. Oscillations of all leukocyte types (other than neutrophils) have been observed in patients with cyclical neutropenia, usually with the same periods. The understanding of cyclical neutropenia has been greatly aided by the discovery that a canine race, the grey collie, gets this congenital disease (with rather shorter periods, in the order of 11 to 16 days).

Another severe disease of the white cells is chronic myelogenous leukemia [19], a cancer of leukocytes. In some cases, chronic myelogenous leukemia also exhibits periodic oscillations in all blood cell counts, with periods falling in the range of 30 to 110 days, but usually about 70-80 days.

These two blood cell diseases are known as periodic hematological diseases. They both originate from the hematopoietic stem cell compartment: a mutation in one hematopoietic stem cell is carried on through successive cell divisions and the disease appears in the bloodstream.

Mathematical modelling of hematopoietic stem cells dynamics has been extensively studied in the past 30 years, with attempts to determine causes leading to a number of periodic hematological diseases. In 1978, Mackey [26] proposed a mathematical model of hematopoietic stem cells dynamics inspired by the works of Lajtha [24] and Burns and Tannock [12]. Formed by a system of two nonlinear delay differential equations, where the delay describes the average cell cycle duration, this model stressed the influence of some factors (such as the apoptotic rate, the introduction rate, the cell cycle duration) playing an important role in the appearance of periodic solutions. Many authors studied properties of the model introduced by Mackey [26] and other models related to this one, in order to understand the role played by hematopoietic stem cells in the stability of hematopoiesis. We refer to Mackey and Rey [28, 29, 30], Mackey and Rudnicki [31, 32], Dyson et al. [15, 16, 17, 18], Pujo-Menjouet and Rudnicki [37], Adimy and Pujo-Menjouet [7], Adimy and Crauste [1, 2] and Adimy et al. [4] for studies of structured models of hematopoiesis, the structure being either age, maturity or age-maturity. We want to point out that we do not consider any structure in the model analyzed in this paper, but these references could be used to improve the present work.

The model of Mackey [26] has been recently analyzed by Pujo-Menjouet and Mackey [36] 
and Pujo-Menjouet et al. [35] in order to prove the existence of long periods oscillations, characterizing situations observed in patients with chronic myelogenous leukemia. Taking into account that all stem cells do not require the same time in cell cycle to divide, Adimy et al. [5, 6, 3] studied models similar to the one proposed by Mackey [26] but with a time delay distributed according to a density with compact support and highlighted the role of a destabilization of the cell cycle duration in the appearance of oscillating solutions with long periods.

In 1998, Haurie et al. [23, 21, 22] published works about cyclical neutropenia, pointing out the main problems and questions arising in its study. They also gave a complete description of the disease. Bernard et al. [9] proposed the first model of leukopoiesis, based on a description of mechanisms involved in cyclical neutropenia. See also [10]. Their model consists of a system of two nonlinear differential equations with two time delays, that describe the cell cycle duration and the time required by a hematopoietic stem cell to produce white blood cells by differentiation. Bernard et al. [9] simplified their model by using a quasi steady-state assumption (so the differentiation rate $k$ in system (2) is assumed to be constant in their model), resulting in a new uncoupled system, whose resolution is equivalent to the one of the model proposed by Mackey [26]. See also [25] for modelling cyclical neutropenia by a system with two delays.

We also mention the recent work of Colijn and Mackey $[13,14]$ which deals with a complete model of hematopoiesis, taking into account the dynamics of the hematopoietic stem cell compartment as well as each of the three blood cell type (red blood cells, white cells and platelets) compartments. The resulting mathematical model is a system of four nonlinear differential equations with six time delays. The authors focused on determining critical model parameters to simulate chronic myelogenous leukemia [13] and cyclical neutropenia [14].

In this paper, we analyze the leukopoiesis model with two delays proposed by Bernard et al. [9] without the simplifying assumption. We want to point out that studying the case with both delays is biologically significant since it makes a clear link between the number of circulating leukocytes and the differentiation of hematopoietic stem cells. Mathematically, the difficulty resides in the presence of two independent delays and the fact that some coefficients in the model equations depend upon these delays. Consequently, the characteristic equation of the linearized system has delay-dependent coefficients. As mentioned by Beretta and Kuang [8], models with delay-dependent coefficients often exhibit very rich dynamics comparing to models with constant coefficients.

When analyzing the stability and bifurcation of planar systems with two delays, we need to study the second degree transcendental polynomials with two delays. The problem of determining the distribution of roots to such polynomials is very complex and there are very few studies on this topic $[11,38,39]$. In this paper, we use an analytical approach proposed by Wei and Ruan [41] and Ruand and Wei [39].

Our work is organized as follows. In the next section, we present the model of leukopoiesis dynamics, a system of two nonlinear delay differential equations with two delays, and investigate the existence of a nontrivial positive steady state. In section 3 , we analyze the asymptotic stability of this steady state. We first linearize the model about the steady state and obtain a second degree exponential polynomial characteristic equation. We determine, successively, conditions for the stability when both time delays are equal to zero, only one delay is equal to zero, and, eventually, when both delays are nonzero. We also establish the existence of a Hopf bifurcation, which destabilizes the entire system and leads to the existence of periodic solutions. In section 4 , we numerically illustrate our results and obtain very rich dynamics of our model that we relate to observations of cyclical neutropenia in patients. We conclude with a discussion. 


\section{The Model}

Hematopoietic stem cells are separated in two distinct compartments: proliferating and nonproliferating cells (see Fig. 1). Proliferating cells are actually in the cell cycle where they particularly perform a growth phase and a phase of DNA synthesis that lead to the cell division, at the end of the phase. After division, the two newborn daughter cells enter immediately a resting phase, also known as $G_{0}$ phase, where they can stay their entire life. This phase is a quiescent stage in the cell cycle with respect to growth and maturation.

During the proliferating phase, hematopoietic stem cells can be eliminated by apoptosis, a specific process aimed to kill deficient cells (a programmed cell death). We denote by $\gamma_{1}$ the rate of apoptosis of hematopoietic stem cells. We assume that the proliferating phase duration is the same for all hematopoietic stem cells and denote it by $\tau_{1}$.

There is a feedback loop between the resting phase and the proliferating phase, which regulates the rate of reentrance in the proliferative compartment from the $G_{0}$ stage. We denote this rate by $\beta$. It is supposed to depend on the quiescent stem cell population $Q(t)$ (see Mackey [26], Pujo-Menjouet and Mackey [36]). More precisely, $\beta$ is a positive monotone decreasing function of $Q$, such that

$$
\lim _{Q \rightarrow \infty} \beta(Q)=0
$$

Typically, $\beta$ is a Hill function given by

$$
\beta(Q)=\frac{\beta_{0} \theta^{n}}{\theta^{n}+Q^{n}} .
$$

The parameter $\beta_{0}$ represents the maximal rate of introduction in the proliferating phase, $\theta$ is the value for which $\beta$ attains half of its maximum value, and $n$ is the sensitivity of the rate of reintroduction. The coefficient $n$ describes the reaction of $\beta$ due to external stimuli, the action of a growth factor for example (some growth factors are known to trigger the introduction of quiescent cells in the proliferating phase).

The differentiation of hematopoietic stem cells takes place in the $G_{0}$ phase. Following divisions, stem cells become committed stem cells, which are programmed to produce one of the three blood cell types. We denote by $k$ the rate of differentiation of hematopoietic stem cells in leukocytes and by $K$ the rate of differentiation in other blood cells (red blood cells and platelets). The latter rate is assumed to be constant, since we do not consider here other populations than hematopoietic stem cells and leukocytes. Note that the rate $K$ may also take some natural mortality into account. According to [9], the rate $k$ describes a negative feedback, similar to the introduction rate $\beta$, and depends on the population of white blood cells $W(t)$. Hence, we assume that $k$ is a positive monotone decreasing function of $W$, which tends to zero as $W$ tends to infinity (see for example (17)).

When a hematopoietic stem cell differentiates in a committed stem cell, a certain number of generations, say $i$, is necessary to produce a leukocyte. We do not take into account the exact number of generations involved in this process, but only (see [9]) the time needed to perform these $i$ generations, that we denote by $\tau_{2}$, coupled to an amplification parameter, denoted by $A$. In fact, $A \approx \alpha 2^{i}$, where $\alpha \in(0,1)$ is a survival rate.

The effective number of divisions $i$ has been estimated to fall in the range of 15-17 [27,34], giving an amplification parameter $A$ about $3-13 \times 10^{4}$, without taking cell death into account. However, in [9], $A$ ranges from 0 to $10^{6}$, corresponding to a generation number $i$ varying between 0 and 20. Moreover, it is noticeable that data vary dramatically between cell types and that some diseases affect the number of divisions [33]. 


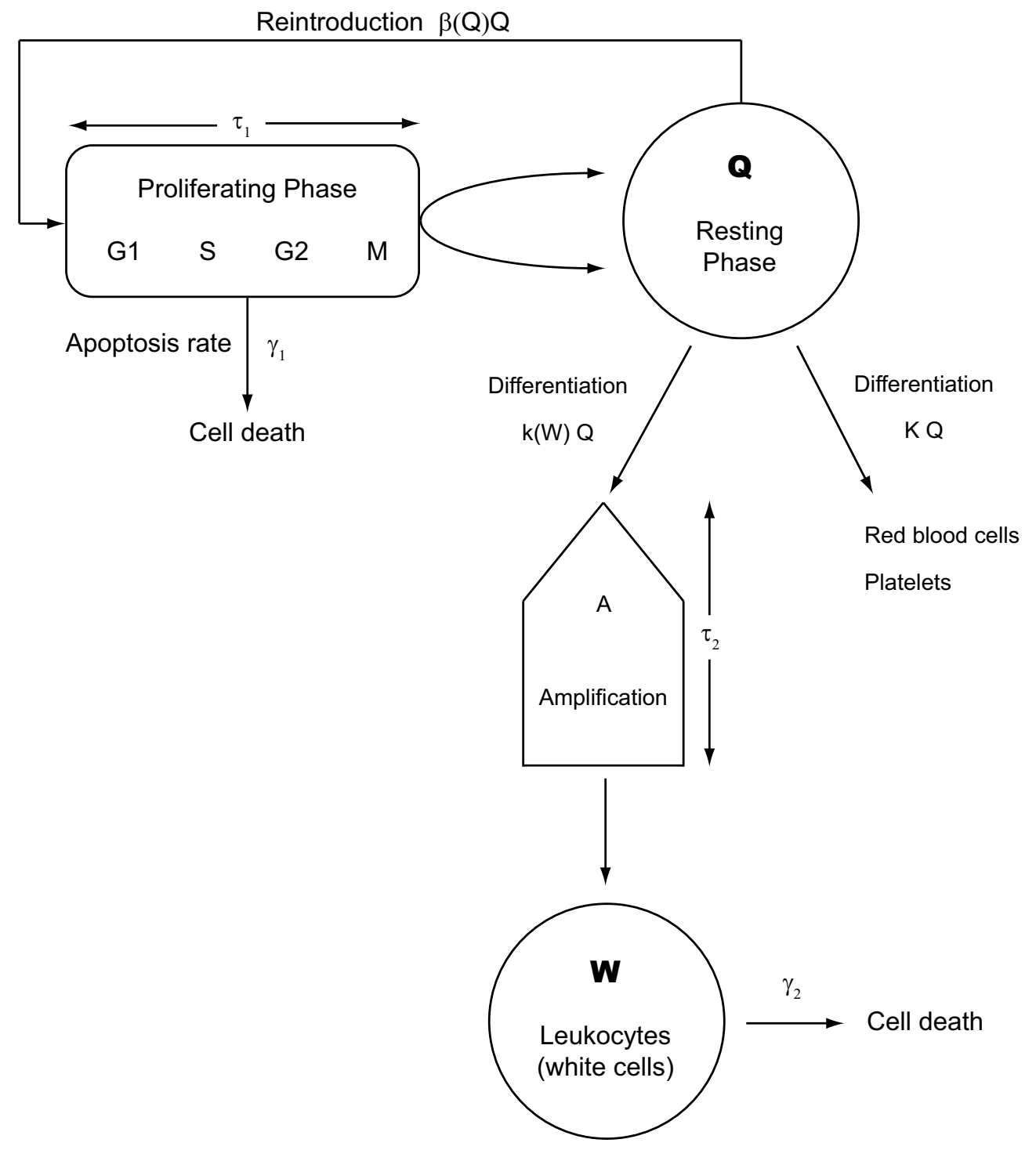

Figure 1: Model of leukopoiesis. The variable $Q$ represents the quiescent cell population in the resting phase. These cells can be introduced in the proliferating phase with a rate $\beta(Q)$ or differentiate in blood cells. In the proliferating phase, cells can die by apoptosis with a rate $\gamma_{1}$, and the proliferating phase duration is $\tau_{1}$. After division of proliferating cells, the two daughter cells immediately enter the resting phase. Resting cells differentiate either in white blood cells (leukocytes) with a rate $k(W)$, depending on the number of white cells $W$, or in red blood cells and platelets with a rate $K$ (that also takes into account natural mortality). Cells in the differentiation pathway are amplified by successive divisions with a factor $A$ (note that cell loss during the differentiation process are included in $A$ ). After a time $\tau_{2}$, differentiating cells become mature white cells. These latter are assumed to disappear with a rate $\gamma_{2}$. 
We want to point out that $\tau_{2}$, the time required to perform the necessary divisions to obtain a white blood cell, should be distributed according to a gamma distribution [9]. Yet, to be able to perform an advanced analysis of our model, we do not make this assumption in this paper.

Denoting by $\gamma_{2}$ the natural mortality rate of white blood cells, our model, proposed by Bernard et al. $[9,10]$, is then the following:

$$
\left\{\begin{aligned}
& \frac{d Q}{d t}=-[K+k(W(t))+\beta(Q(t))] Q(t) \\
&+2 e^{-\gamma_{1} \tau_{1}} \beta\left(Q\left(t-\tau_{1}\right)\right) Q\left(t-\tau_{1}\right), \\
& \frac{d W}{d t}=-\gamma_{2} W(t)+A k\left(W\left(t-\tau_{2}\right)\right) Q\left(t-\tau_{2}\right) .
\end{aligned}\right.
$$

One can note that the second term in the right hand side of the $Q$ equation in (2) accounts for cells produced by division at the end of the proliferating phase. These cells are in fact quiescent cells introduced one generation earlier in the proliferating phase. The factor 2 describes the division of each cell into two daughter cells (see [26]).

The functions $\beta$ and $k$ are bounded. Assuming that they are locally Lipschitz continuous (which is always the case for smooth functions, say continuously differentiable; in particular, for $\beta$ and $k$ given by (17) in Section 4), one can easily check, from Hale and Verduyn Lunel [20], that system (2) has a unique continuous solution $(Q(t), W(t))$, which is well-defined for all $t \geq 0$ and for a continuous initial condition. Moreover, we easily see that, for nonnegative initial conditions, the solutions of (2) remain nonnegative for $t \geq 0$. Indeed, let $T>0$ be such that $Q(t)>0$ for $t<T$ with $Q(T)=0$. Then, from (2), we have

$$
\frac{d Q}{d t}(T)=2 e^{-\gamma_{1} \tau_{1}} \beta\left(Q\left(T-\tau_{1}\right)\right) Q\left(T-\tau_{1}\right)>0,
$$

and the result follows. The same reasoning holds for $W(t)$.

Now, let us turn our considerations on the existence of steady states for system (2). Steady states of $(2)$ are stationary solutions $\left(Q^{*}, W^{*}\right)$ of $(2)$, that is,

$$
\left\{\begin{aligned}
{\left[K+k\left(W^{*}\right)+\beta\left(Q^{*}\right)\right] Q^{*} } & =2 e^{-\gamma_{1} \tau_{1}} \beta\left(Q^{*}\right) Q^{*}, \\
\gamma_{2} W^{*} & =A k\left(W^{*}\right) Q^{*} .
\end{aligned}\right.
$$

Firstly, notice that $(0,0)$ is always a steady state of $(2)$. It describes the extinction of the cell populations.

Searching for nonzero steady states of $(2)$, that is $\left(Q^{*}, W^{*}\right) \neq(0,0)$, we must solve

$$
\begin{aligned}
K+k\left(W^{*}\right) & =\left(2 e^{-\gamma_{1} \tau_{1}}-1\right) \beta\left(Q^{*}\right), \\
\gamma_{2} W^{*} & =A k\left(W^{*}\right) Q^{*} .
\end{aligned}
$$

We have the following result, whose proof is detailed in Appendix A.

Proposition 2.1. Assume that

$$
\left(2 e^{-\gamma_{1} \tau_{1}}-1\right) \beta(0)>k(0)+K
$$

and that the function $Q \mapsto Q \beta(Q)$ is decreasing on the interval $\left(Q_{0}, Q_{1}\right)$, where

$$
Q_{0}=\beta^{-1}\left(\frac{k(0)+K}{2 e^{-\gamma_{1} \tau_{1}}-1}\right) \quad \text { and } \quad Q_{1}=\beta^{-1}\left(\frac{K}{2 e^{-\gamma_{1} \tau_{1}}-1}\right) .
$$

Then system (2) has a unique nontrivial positive steady state $\left(Q^{*}, W^{*}\right)$, a solution of system (3)-(4). 
Remark 1. (i) One can note that, with $\beta$ given by (1), the function $Q \mapsto Q \beta(Q)$ is decreasing on the interval $\left[\theta /(n-1)^{1 / n},+\infty\right)$ provided that $n>1$, and $\left(Q_{0}, Q_{1}\right) \subset\left[\theta /(n-1)^{1 / n},+\infty\right)$ if and only if

$$
n>\frac{\left(2 e^{-\gamma_{1} \tau_{1}}-1\right) \beta_{0}}{\left(2 e^{-\gamma_{1} \tau_{1}}-1\right) \beta_{0}-k(0)-K} .
$$

(ii) Recall that $(0,0)$ is always a steady state of $(2)$.

(iii) When condition (5) no longer holds, we cannot conclude the uniqueness - or even the existence - of a nontrivial steady state of (2). If $K \geq\left(2 e^{-\gamma_{1} \tau_{1}}-1\right) \beta(0)$, then there is no other steady state than $(0,0)$. Yet, if $k(0)+K \geq\left(2 e^{-\gamma_{1} \tau_{1}}-1\right) \beta(0)>K$, system (2) may exhibit several steady states, depending on the values of the first derivatives of $\beta$ and $k$.

In the next section, we focus on the asymptotic stability of the unique nontrivial steady state of system $(2)$, namely $\left(Q^{*}, W^{*}\right)$, defined in Proposition 2.1. To that aim, we linearize (2) about $\left(Q^{*}, W^{*}\right)$ and determine the associated characteristic equation.

\section{Asymptotic Stability}

Throughout this section, we assume that condition (5) holds and that the mapping $Q \mapsto$ $Q \beta(Q)$ is decreasing on $\left(Q_{0}, Q_{1}\right)$, with $Q_{0}$ and $Q_{1}$ defined in (6). Thus, the unique nontrivial steady state $\left(Q^{*}, W^{*}\right)$, with $Q^{*}, W^{*}>0$, of $(2)$ is well defined from Proposition 2.1 by (3)-(4). Moreover, we assume that $\beta$ and $k$ are $\mathcal{C}^{1}$ functions.

We first linearize system $(2)$ around $\left(Q^{*}, W^{*}\right)$ and deduce the characteristic equation.

\subsection{Linearization and Characteristic Equation}

In the following, we will use the notations

$$
\begin{aligned}
k^{*} & :=k\left(W^{*}\right), \\
\beta^{*} & :=\beta\left(Q^{*}\right)+Q^{*} \beta^{\prime}\left(Q^{*}\right) .
\end{aligned} \quad \overline{k^{*}}:=Q^{*} k^{\prime}\left(W^{*}\right),
$$

Setting $q(t)=Q(t)-Q^{*}$ and $w(t)=W(t)-W^{*}$, the linearized system of (2) about $\left(Q^{*}, W^{*}\right)$ is then given by

$$
\left\{\begin{aligned}
\frac{d q}{d t} & =-\left[K+k^{*}+\beta^{*}\right] q(t)-\overline{k^{*}} w(t)+2 e^{-\gamma_{1} \tau_{1}} \beta^{*} q\left(t-\tau_{1}\right), \\
\frac{d w}{d t} & =-\gamma_{2} w(t)+A\left[k^{*} q\left(t-\tau_{2}\right)+\overline{k^{*}} w\left(t-\tau_{2}\right)\right] .
\end{aligned}\right.
$$

Define

$$
\mathcal{A}_{0}:=\left(\begin{array}{cc}
-\left(K+k^{*}+\beta^{*}\right) & -\overline{k^{*}} \\
0 & -\gamma_{2}
\end{array}\right), \quad \mathcal{A}_{1}:=\left(\begin{array}{cc}
2 e^{-\gamma_{1} \tau_{1}} \beta^{*} & 0 \\
0 & 0
\end{array}\right)
$$

and

$$
\mathcal{A}_{2}:=A\left(\begin{array}{cc}
0 & 0 \\
k^{*} & \overline{k^{*}}
\end{array}\right)
$$

The characteristic equation associated with (7) is given by

$$
\operatorname{det}\left(\lambda I-\mathcal{A}_{0}-e^{-\lambda \tau_{1}} \mathcal{A}_{1}-e^{-\lambda \tau_{2}} \mathcal{A}_{2}\right)=0 .
$$


After simplifications, this equation becomes

$$
\begin{aligned}
\lambda^{2}+\left(K+k^{*}+\beta^{*}+\gamma_{2}\right) \lambda+\left(K+k^{*}+\beta^{*}\right) \gamma_{2} & \\
-2 e^{-\gamma_{1} \tau_{1}} \beta^{*}\left(\lambda+\gamma_{2}\right) e^{-\lambda \tau_{1}}-A \overline{k^{*}}\left(\lambda+\beta^{*}+K\right) e^{-\lambda \tau_{2}} & \\
+2 A e^{-\gamma_{1} \tau_{1}} \beta^{*} \overline{k^{*}} e^{-\lambda\left(\tau_{1}+\tau_{2}\right)} & =0 .
\end{aligned}
$$

This is a second degree exponential polynomial in $\lambda$. The local asymptotic stability analysis of the steady state $\left(Q^{*}, W^{*}\right)$ is performed through the study of the sign of the real parts of roots of $(8)$. We recall that $\left(Q^{*}, W^{*}\right)$ is locally asymptotically stable if and only if all roots of (8) have negative real parts, and its stability can only be lost if roots cross the vertical axis, that is if purely imaginary roots appear.

Because of the presence of two different delays, $\tau_{1}$ and $\tau_{2}$, in equation (8), the analysis of the sign of the real parts of eigenvalues is very complicated, and a direct approach cannot be considered. We will use a method consisting of determining the stability of the steady state when one delay is equal to zero, and, using similar analytic arguments as in [39], we will deduce conditions for the stability of the steady state when both time delays are nonzero.

Note also that the steady state $\left(Q^{*}, W^{*}\right)$ implicitly depends on the time delay $\tau_{1}$, through (3)-(4). Therefore, coefficients of the characteristic equation (8) depend, explicitly (the term with $e^{-\gamma_{1} \tau_{1}}$ ) or implicitly, upon the delay $\tau_{1}$. This particularity adds a complexity to the resolution of (8).

\subsection{The case $\tau_{1}=\tau_{2}=0$.}

Assume that $\tau_{1}=\tau_{2}=0$. Then, the characteristic equation (8) is written as a second degree polynomial equation

$$
\lambda^{2}+\left(K+k^{*}+\gamma_{2}-\beta^{*}-A \overline{k^{*}}\right) \lambda+\left(K+k^{*}-\beta^{*}\right) \gamma_{2}-A \overline{k^{*}}\left(K-\beta^{*}\right)=0 .
$$

From the Routh-Hurwith criterion, all eigenvalues of (9) have negative real parts if and only if

$$
K+k^{*}+\gamma_{2}>\beta^{*}+A \overline{k^{*}}
$$

and

$$
\left(K+k^{*}-\beta^{*}\right) \gamma_{2}>A \overline{k^{*}}\left(K-\beta^{*}\right) .
$$

From (3), the steady state $\left(Q^{*}, W^{*}\right)$ of $(2)$ satisfies,

$$
K+k\left(W^{*}\right)=\beta\left(Q^{*}\right) .
$$

Since $\beta^{*}=\beta\left(Q^{*}\right)+Q^{*} \beta^{\prime}\left(Q^{*}\right)$, condition (10) is then equivalent to

$$
\gamma_{2}>Q^{*} \beta^{\prime}\left(Q^{*}\right)+A \overline{k^{*}}
$$

The functions $\beta$ and $k$ are decreasing, so $\beta^{\prime}\left(Q^{*}\right) \leq 0$ and $\overline{k^{*}} \leq 0$. Consequently, (11) holds true, and (10) is satisfied. We can then conclude to the asymptotic stability of $\left(Q^{*}, W^{*}\right)$ when $\tau_{1}=\tau_{2}=0$ in the next proposition.

Proposition 3.1. Assume that

$$
\left(H_{1}\right) \quad\left(K+k^{*}-\beta^{*}\right) \gamma_{2}>A \overline{k^{*}}\left(K-\beta^{*}\right) .
$$

Then all eigenvalues of (9) have negative real parts, and the steady state $\left(Q^{*}, W^{*}\right)$ of system (2) is locally asymptotically stable.

One can notice that, for example, when $\beta^{*}$ is negative, assumption $\left(H_{1}\right)$ holds true, since $k^{*}>0$ and $\overline{k^{*}} \leq 0$. For values of the parameters taken from the literature (see Section 4 ), one can see that condition $\left(H_{1}\right)$ is fulfilled. 


\subsection{The case $\tau_{1}=0$ and $\tau_{2}>0$.}

We now consider the case $\tau_{1}=0$ and $\tau_{2}>0$. Our choice is motivated by the following biological reasons: the time delay $\tau_{1}$ represents the average cell cycle duration of hematopoietic stem cells whereas $\tau_{2}$ represents the time needed by hematopoietic cells to differentiate in white blood cells. This latter is longer than $\tau_{1}$ (about one week, when the cell cycle duration usually falls in the range of 1 to 4 days). Since the final result we will obtain gives the stability of $\left(Q^{*}, W^{*}\right)$ for values of $\tau_{1}$ less than the ones of $\tau_{2}$, we prefer to choose $\tau_{1}=0$ in this section. Also, since we want to estimate the critical delay values, then, with $\tau_{1}=0$, instead of using the results of Beretta and Kuang [8], we analyze the characteristic equation directly.

Setting $\tau_{1}=0$ in (8), the characteristic equation becomes

$$
\lambda^{2}+b_{1} \lambda+b_{2}+\left[b_{3} \lambda+b_{4}\right] e^{-\lambda \tau_{2}}=0,
$$

where

$$
\begin{array}{ll}
b_{1}=K+k^{*}+\gamma_{2}-\beta^{*}, & b_{3}=-A \overline{k^{*}}, \\
b_{2}=\left(K+k^{*}-\beta^{*}\right) \gamma_{2}, & b_{4}=-A \overline{k^{*}}\left(K-\beta^{*}\right) .
\end{array}
$$

We mention that, from $(3)-(4)$, the steady state $\left(Q^{*}, W^{*}\right)$ is then defined by

$$
\begin{aligned}
& \beta\left(Q^{*}\right)=K+k\left(W^{*}\right), \\
& \gamma_{2} W^{*}=A k\left(W^{*}\right) Q^{*}
\end{aligned}
$$

One can notice that, using (13), we obtain

$$
b_{1}=\gamma_{2}-Q^{*} \beta^{\prime}\left(Q^{*}\right)>0, \quad b_{2}=-\gamma_{2} Q^{*} \beta^{\prime}\left(Q^{*}\right)>0, \quad b_{3}>0 .
$$

Moreover, from the reasoning in the previous section, $b_{2}+b_{4}>0$.

Since (12) reduces to $(9)$ when $\tau_{2}=0$, under assumption $\left(H_{1}\right)$ the steady state $\left(Q^{*}, W^{*}\right)$ is locally asymptotically stable when $\tau_{2}=0$ (see Proposition 3.1). Consequently, with assumption $\left(H_{1}\right)$, when $\tau_{2}$ increases, the stability of the steady state can only be lost if pure imaginary roots appear. Hence we look for purely imaginary roots $\lambda= \pm i \omega, \omega \in \mathbb{R}$, of (12).

If $i \omega$ is a purely imaginary root of (12), then separating real and imaginary parts, $\omega$ satisfies

$$
\begin{aligned}
\cos \left(\omega \tau_{2}\right) & =\frac{\left(b_{4}-b_{1} b_{3}\right) \omega^{2}-b_{2} b_{4}}{b_{3}^{2} \omega^{2}+b_{4}^{2}} \\
\sin \left(\omega \tau_{2}\right) & =\frac{b_{3} \omega^{3}+\left(b_{1} b_{4}-b_{2} b_{3}\right) \omega}{b_{3}^{2} \omega^{2}+b_{4}^{2}} .
\end{aligned}
$$

Note that $\omega=0$ is not a solution of (14)-(15) since $b_{2}+b_{4}>0$.

One can notice that if $\omega$ is a solution of (14)-(15), then so is $-\omega$. Hence, in the following, we only look for positive solutions $\omega$ of (14)-(15).

Adding the squares of both hand sides of equations (14) and (15), it follows that $\omega$ must be a root of the following equation

$$
\omega^{4}+\left(b_{1}^{2}-2 b_{2}-b_{3}^{2}\right) \omega^{2}+b_{2}^{2}-b_{4}^{2}=0 .
$$

Set

$$
F(X)=X^{2}+\left(b_{1}^{2}-2 b_{2}-b_{3}^{2}\right) X+b_{2}^{2}-b_{4}^{2}
$$


and consider the assumption

$$
\left(H_{2}\right) \quad b_{2}^{2}-b_{4}^{2}<0 \quad \text { or } \quad\left(b_{1}^{2}-2 b_{2}-b_{3}^{2}\right)^{2} \geq 4\left(b_{2}^{2}-b_{4}^{2}\right) \geq 0>b_{1}^{2}-2 b_{2}-b_{3}^{2} .
$$

The function $F$ has positive roots if and only if $\left(H_{2}\right)$ holds. Denote (without loss of generality) by $X_{l}, l=1,2$, the positive roots of $F$, and set

$$
\omega_{l}=\sqrt{X_{l}}
$$

Notice that the unique solution $\theta \in[0,2 \pi]$ of

$$
\cos (\theta)=\frac{\left(b_{4}-b_{1} b_{3}\right) \omega_{l}^{2}-b_{2} b_{4}}{b_{3}^{2} \omega_{l}^{2}+b_{4}^{2}}, \quad \sin (\theta)=\frac{b_{3} \omega_{l}^{3}+\left(b_{1} b_{4}-b_{2} b_{3}\right) \omega_{l}}{b_{3}^{2} \omega_{l}^{2}+b_{4}^{2}},
$$

is $\theta=\arccos \left(\left(b_{4}-b_{1} b_{3}\right) \omega_{l}^{2}-b_{2} b_{4} / b_{3}^{2} \omega_{l}^{2}+b_{4}^{2}\right)$ if $\sin (\theta)>0$, that is if $b_{3} \omega_{l}^{2}+b_{1} b_{4}-b_{2} b_{3}>0$, and $\theta=2 \pi-\arccos \left(\left(b_{4}-b_{1} b_{3}\right) \omega_{l}^{2}-b_{2} b_{4} / b_{3}^{2} \omega_{l}^{2}+b_{4}^{2}\right)$ if $b_{3} \omega_{l}^{2}+b_{1} b_{4}-b_{2} b_{3} \leq 0$.

We define two sequences $\left\{\tau_{2, l}^{1, j}\right\}$ and $\left\{\tau_{2, l}^{2, j}\right\}$, for $l=1,2$ and $j \in \mathbb{N}$, by

$$
\tau_{2, l}^{1, j}=\frac{1}{\omega_{l}}\left[\arccos \left(\frac{\left(b_{4}-b_{1} b_{3}\right) \omega_{l}^{2}-b_{2} b_{4}}{b_{3}^{2} \omega_{l}^{2}+b_{4}^{2}}\right)+2 j \pi\right]
$$

and

$$
\tau_{2, l}^{2, j}=\frac{1}{\omega_{l}}\left[2 \pi-\arccos \left(\frac{\left(b_{4}-b_{1} b_{3}\right) \omega_{l}^{2}-b_{2} b_{4}}{b_{3}^{2} \omega_{l}^{2}+b_{4}^{2}}\right)+2 j \pi\right] .
$$

The proof of the following lemma can be found in Appendix B.

Lemma 3.1. Let $\tau_{2, l}^{*}$ denote an element of either the sequence $\left\{\tau_{2, l}^{1, j}\right\}$ or $\left\{\tau_{2, l}^{2, j}\right\}$, associated with $\omega_{l}$ (that is $\tau_{2, l}^{*}=\tau_{2, l}^{1, j}$ or $\tau_{2, l}^{*}=\tau_{2, l}^{2, j}$ ). For $\tau_{2}=\tau_{2, l}^{*}$, the characteristic equation (12) has a pair of simple conjugate pure imaginary roots $\pm i \omega_{l}$, satisfying

$$
\operatorname{sign}\left\{\left.\frac{d R e(\lambda)}{d \tau_{2}}\right|_{\tau=\tau_{2, l}^{*}}\right\}=\operatorname{sign}\left\{F^{\prime}\left(\omega_{l}^{2}\right)\right\} .
$$

Based on the above analysis, we conclude, in the next theorem, the stability of the steady state $\left(Q^{*}, W^{*}\right)$ of $(2)$ when $\tau_{1}=0$.

Theorem 3.1. Assume that $\left(H_{1}\right)$ and $\left(H_{2}\right)$ hold true. Let

$$
\tau_{2}^{*}=\tau_{2, l_{0}}^{i_{0}, j_{0}}:=\min _{l=1,2 ; j \in \mathbb{N}}\left\{\tau_{2, l}^{1, j} ; \tau_{2, l}^{2, j}\right\}
$$

Then the steady state $\left(Q^{*}, W^{*}\right)$ of (2) is locally asymptotically stable when $\tau_{2}<\tau_{2}^{*}$ and a Hopf bifurcation occurs at $\left(Q^{*}, W^{*}\right)$ when $\tau_{2}=\tau_{2}^{*}$ if and only if $F^{\prime}\left(\omega_{l_{0}}^{2}\right)>0$.

One can check, in Section 4, that for values of the parameters taken from the literature condition $\left(H_{2}\right)$ is easily fulfilled.

\subsection{The case $\tau_{1}, \tau_{2}>0$.}

We now return to the study of equation (8) with $\tau_{1}, \tau_{2}>0$. In order to study the local stability of the positive steady state $\left(Q^{*}, W^{*}\right)$ of $(2)$, we first prove a result regarding the sign of the real parts of characteristic roots of (8) in the next lemma. 
Lemma 3.2. If all roots of equation (12) have negative real parts for $\tau_{2}>0$, then there exists $a \tau_{1}^{*}\left(\tau_{2}\right)>0$ such that all roots of equation (8) have negative real parts when $\tau_{1}<\tau_{1}^{*}\left(\tau_{2}\right)$.

Proof. Assume that equation (12) has no root with nonnegative real part for $\tau_{2}>0$. Thus, equation (8) with $\tau_{1}=0$ and $\tau_{2}>0$ has no root with nonnegative real part.

Regard $\tau_{1}$ as a parameter. Clearly, the left hand side of equation (8) is analytic in $\lambda$ and $\tau_{1}$. Following Theorem 2.1 of [39], as $\tau_{1}$ varies, the sum of the multiplicity of zeros of the left hand side of equation (8) in the open right half-plane can only change if a zero appears on or crosses the imaginary axis.

Since equation (8) with $\tau_{1}=0$ has no root with nonnegative real part, there exists a $\tau_{1}^{*}\left(\tau_{2}\right)>0$ such that all roots of equation (8) with $\tau_{1}<\tau_{1}^{*}\left(\tau_{2}\right)$ have negative real parts.

Using Theorem 3.1, we have the following theorem about the asymptotic stability of the positive steady state of (2).

Theorem 3.2. Assume that $\left(H_{1}\right)$ and $\left(H_{2}\right)$ hold true. Let $\tau_{2}^{*}$ be defined as in Theorem 3.1. Then for any $\tau_{2} \in\left[0, \tau_{2}^{*}\right)$, there exists a $\tau_{1}^{*}\left(\tau_{2}\right)>0$ such that the positive steady state $\left(Q^{*}, W^{*}\right)$ of system (2) is locally asymptotically stable when $\tau_{1} \in\left[0, \tau_{1}^{*}\left(\tau_{2}\right)\right)$.

Proof. From Theorem 3.1, it follows that all roots of equation (12) have negative real parts when $\tau_{2} \in\left[0, \tau_{2}^{*}\right)$. We conclude with Lemma 3.2 .

Remark 2. An overview of the behavior of the steady state $\left(Q^{*}, W^{*}\right)$ (stable or unstable), which is more complete than the result given by Theorem 3.2, is shown on Fig. 2. It should be helpful in understanding the effect of the two delays on the stability of the steady state.

In the next section we numerically illustrate the stability result obtained in Theorem 3.2, as well as the existence of periodic solutions through the Hopf bifurcation.

\section{Periodic Oscillations: Numerical Illustrations}

In this section, we illustrate the different stability results obtained in the previous section, mainly in Theorem 3.2. We also focus on periodic solutions appearing through a Hopf bifurcation, and we relate oscillating solutions to behaviors observed with cyclical neutropenia $[9]$.

First, let us choose the negative feedback functions $\beta$ and $k$ as proposed by Bernard et al. [9], that is, monotone decreasing positive functions defined by

$$
\beta(Q)=\frac{\beta_{0}}{1+Q^{n}} \quad \text { and } \quad k(W)=\frac{k_{0}}{1+W^{m}} .
$$

Most values of the different parameters involved in system (2) can be found in the literature. The values of the above parameters $\beta_{0}, k_{0}, n$ and $m$ are given by Mackey [26], Pujo-Menjouet and Mackey [36] and Bernard et al. [9, 10] as follows:

$$
\beta_{0}=1.77 \text { days }^{-1}, \quad k_{0}=0.1 \text { days }^{-1}, \quad n=3, \quad m=2 .
$$

Using [9] and [26, 27], the mortality rates $\gamma_{1}$ and $\gamma_{2}$, and the differentiation rate $K$, are set to

$$
\gamma_{1}=0.1 \text { days }^{-1}, \quad \gamma_{2}=2.4 \text { days }^{-1}, \quad K=0.02 \text { days }^{-1} .
$$




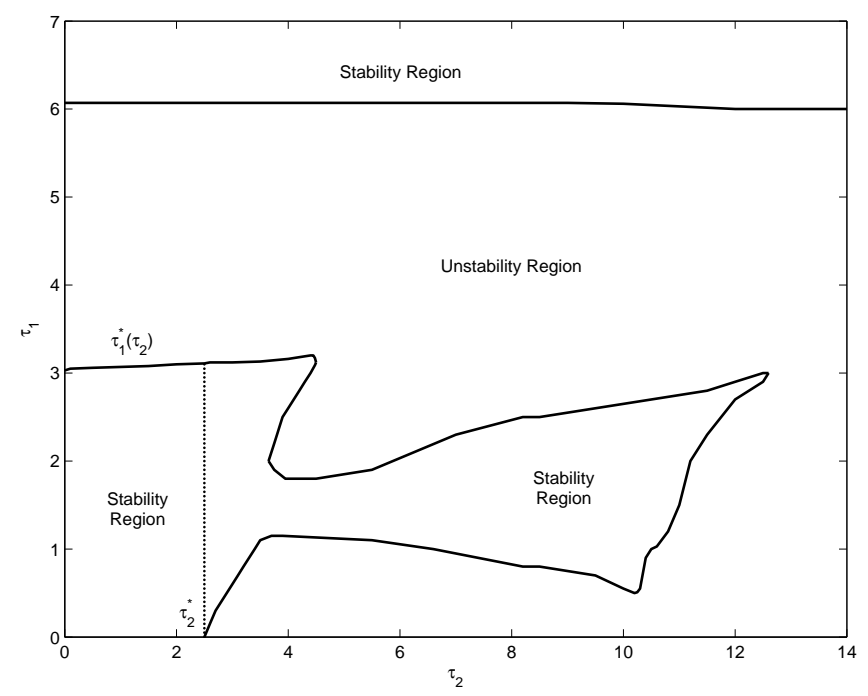

Figure 2: Stability diagram. Stability of the steady state $\left(Q^{*}, W^{*}\right)$ of system $(2)$ is displayed as a function of $\tau_{1}$ and $\tau_{2}$. The full lines are the boundaries between stable and unstable regions. This diagram was obtained using the values mentioned in Section 4, in (18) and (19), with $\mathrm{A}=20$. In this case, the steady state $\left(Q^{*}, W^{*}\right)$ of system (2) no longer exists when $\tau_{1} \geq 7$ days, and the critical value $\tau_{2}^{*}$ obtained in Theorem 3.1 equals 2.5 days and is indicated on the figure. The more biologically relevant area for $\tau_{1}$ and $\tau_{2}$ corresponds to small values of the two parameters, close to one day. It is a stable region given by Theorem 3.2 (bounded by the vertical dotted line $\tau_{2}=\tau_{2}^{*}$ and the curve $\tau_{1}=\tau_{1}^{*}\left(\tau_{2}\right)$ ). However, one can note very rich dynamics of the steady state. As $\tau_{1}$ (respectively $\tau_{2}$ ) increases, a finite number of stability switches may occur. 


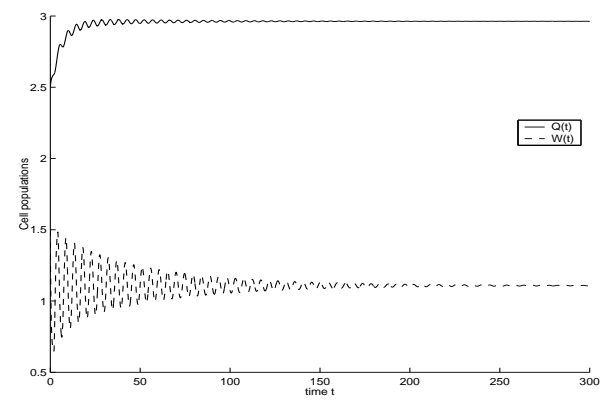

(a)

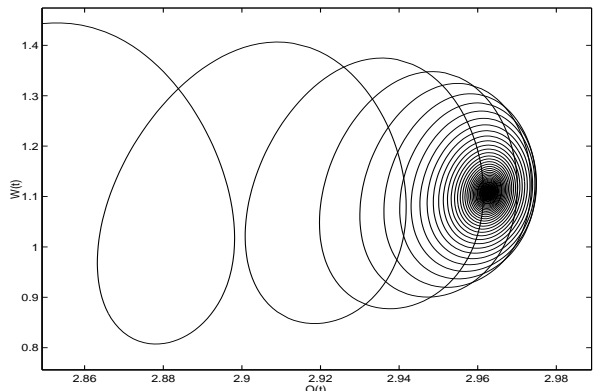

(b)

Figure 3: Asymptotic stability. The steady state $\left(Q^{*}, W^{*}\right)$ of $(2)$ is locally asymptotically stable, even though damped oscillations can be observed. In figure (a), the solid line is for the hematopoietic stem cell population $Q(t)$ and the dashed one is for the white blood cell population $W(t)$. In figure (b), the solutions are drawn in the $(Q, W)$-plane. Parameters are given by (18) and (19), with $A=20$. The time delays are $\tau_{1}=0.05$ and $\tau_{2}=2$ days.

Note that, with values in (18) and (19), (5) holds true if $\tau_{1} \leq 6.27$ days and the function $Q \mapsto Q \beta(Q)$ is decreasing on $\left(Q_{0}, Q_{1}\right)$ (see $\left.(6)\right)$ if $\tau_{1} \leq 5.96$ days (cf. Remark 1 (i)). Hence, the steady state $\left(Q^{*}, W^{*}\right)$ of $(2)$ exists and is unique for $\tau_{1} \leq 5.96$ days. Finally, as described in [9], we choose $A=20$. With this value, damped oscillations can be observed in (2).

We mention that all numerical simulations presented in this section have been done with dde23 [40].

For values of $\tau_{2}$ less than the critical value for which a Hopf bifurcation occurs (namely, $\tau_{2}^{*}$, as stated in Theorem 3.1$)$, then $\left(Q^{*}, W^{*}\right)$ must be asymptotically stable for small values of $\tau_{1}$ (see Theorem 3.2). This is represented in Fig. 3. We observe damped oscillations about the steady state, although it is asymptotically stable.

Simple computations indicate that the Hopf bifurcation occurs at $\left(Q^{*}, W^{*}\right)$ when $\tau_{1}=0$ for $\tau_{2}$ about 2.5 days. For values of $\tau_{2}$ greater than the critical value, we observe oscillating solutions, with periods falling in the range of 10 to 15 days (see Fig. 4). One can note that the white blood cell population $W(t)$ oscillates with larger amplitudes than the hematopoietic stem cell population (this difference seems to be due to the amplification of the leukocyte population, induced in the model by the coefficient $A)$. The steady state $\left(Q^{*}, W^{*}\right)$ of $(2)$ is then unstable.

Even though the periods of the oscillations in Fig. 4 are quite short, they can be related to the ones often observed in periodic hematological diseases. For example, in cyclical neutropenia, the number of white blood cells usually decreases every 3 weeks in human and 2 weeks in dogs.

When $\tau_{2}$ keeps on increasing far from the critical value, we obtain oscillations with longer periods (see Fig. 4.b), about 15 days. These oscillations describe situations encountered with periodic hematological diseases, such as cyclical neutropenia. We can notice, for example, that the number of white blood cell decreases to very low values every 15 days. This characterizes cyclical neutropenia [21].

Longer periods can be observed, about 20 to 25 days for both populations, when $\tau_{1}$, the cell cycle duration, is destabilized and increases. In Fig. 5.a are drawn solutions of system (2) when $\tau_{1}=3$ days and $\tau_{2}=7$ days, which seem to be reasonable estimations of these durations. One can observe oscillations similar to the ones obtained in [9], while 

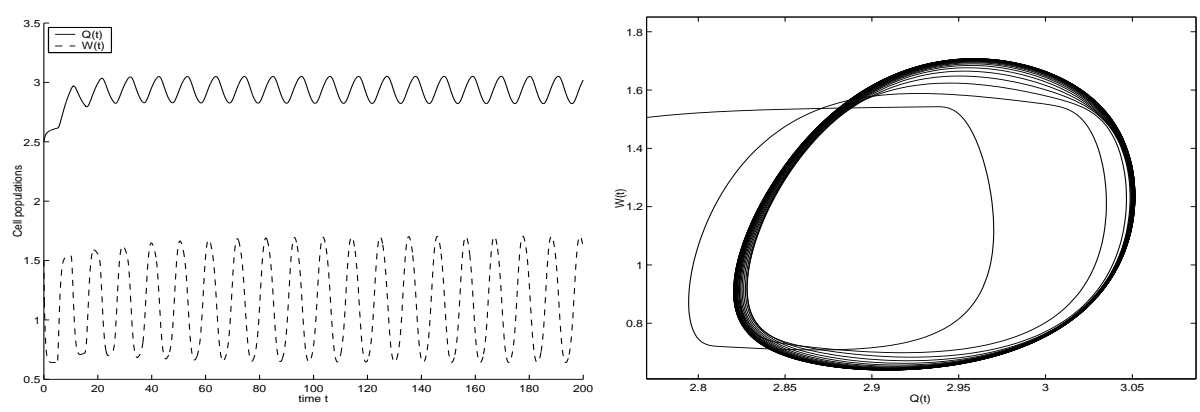

(a)
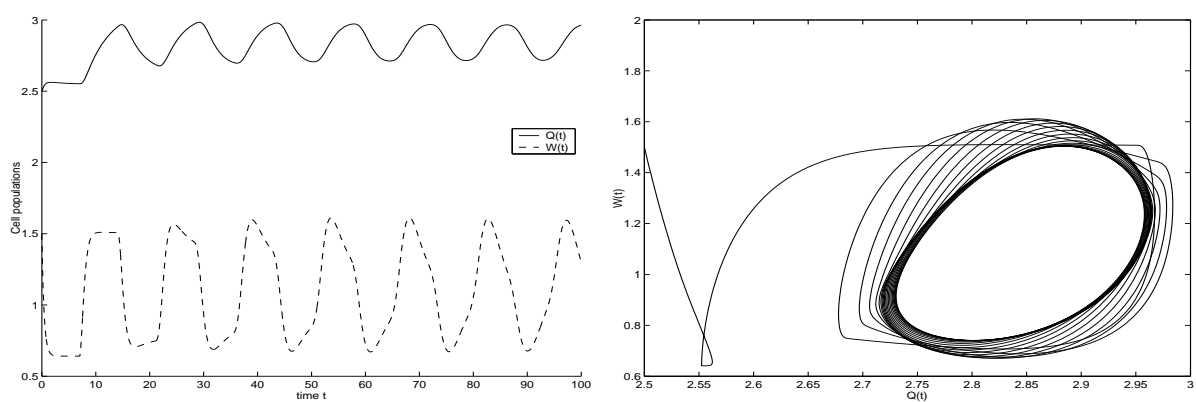

(b)

Figure 4: Oscillating solutions with $\tau_{1}$ close to zero. Parameters are given by (18) and (19), with $A=20$. The time delays are $\tau_{1}=0.05$ and (a) $\tau_{2}=5$ days and (b) $\tau_{2}=7$ days. Oscillating solutions appear with periods about (a) 10 days for the white blood cell population (the dashed line) as well as for the hematopoietic stem cell population (solid line), and (b) 15 days for the two cell populations, with very low values attained by the white blood cell count, as observed in cyclical neutropenia. The steady state $\left(Q^{*}, W^{*}\right)$ of $(2)$ is unstable in both cases. Note that the time scales are different from one picture to an other. 
describing cyclical neutropenia, with longer periods. This behavior remains the same when $\tau_{1}$ increases (see Fig. 5.b and 5.c), up to 5 days, describing a destabilization of the cell cycle duration that is suspected to produce cyclical neutropenia [9]. Oscillating solutions are observed, with longer periods (up to 50 days), and ripples can be observed in Fig. 5.c. This phenomenon has already been noticed by Pujo-Menjouet and Mackey [36]. It seems that a strong perturbation of the cell cycle duration results in a destabilization of the entire process of blood production (in our case, of white blood cell production), with strange observable behaviors of the populations. One can note, for example, the shape of the attractors in the $(Q, W)$-planes in Fig. 5.

\section{Discussion}

In order to understand the population dynamics of the white blood cells in cyclical neutropenia, [9] proposed a leukopoiesis model with two delays. The characteristic equation of the linearized system around the positive equilibrium is a second degree exponential polynomial with delay-dependent coefficients. The authors mentioned in [9] that "it is not possible to study the roots of this equation by means of analytical tools alone". However, in this paper, we carried out a rigorous analysis of the stability of the model proposed by Bernard et al. [9], which improves the analysis presented in [9].

To analyze the characteristic equation with two delays, we first focused on the case when one of the delay, $\tau_{1}$, equals zero and obtained a critical value for the delay $\tau_{2}$ : when $\tau_{2}<\tau_{2}^{*}$ all roots of the characteristic equation have negative parts and when $\tau_{2}=\tau_{2}^{*}$ purely imaginary roots appear. Then we assumed that $\tau_{2}<\tau_{2}^{*}$ and considered the delay $\tau_{1}$. We showed that there is a $\tau_{1}^{*}\left(\tau_{2}\right)$ such that all roots of the characteristic equation have negative real parts when $\tau_{1} \in\left[0, \tau_{1}^{*}\left(\tau_{2}\right)\right)$. Consequently, we obtained stability results for the leukopoiesis model with two independent delays and proved the existence of a Hopf bifurcation that leads to the appearance of periodic solutions.

Oscillating solutions, in the frame of blood cell production models, have often been related to the so-called periodic hematological diseases [21, 26] since a variety of diseases affect blood cells and are characterized by oscillations of these cells. Numerical simulations of our model demonstrated periodic solutions which fit well with oscillations usually observed in cyclical neutropenia $[9,21]$. We obtained oscillations with quite long periods (in the order of 25 to 50 days) for reasonable parameters, in keeping with parameters found in the literature.

The numerical results in Fig. 5 also indicate that our model may have rich dynamics, with very long oscillations and period doubling oscillating solutions, or ripples, as noticed for a simpler model by Pujo-Menjouet and Mackey [36]. A further analysis could help in understanding the complicated dynamics of the blood cell production system and we leave it to future consideration.

\section{Acknowledgements}

Works of Mostafa Adimy and Fabien Crauste were partially supported by the ANUBIS project, INRIA-Futurs. Research of Shigui Ruan was partially supported by the NSF and the University of Miami. 

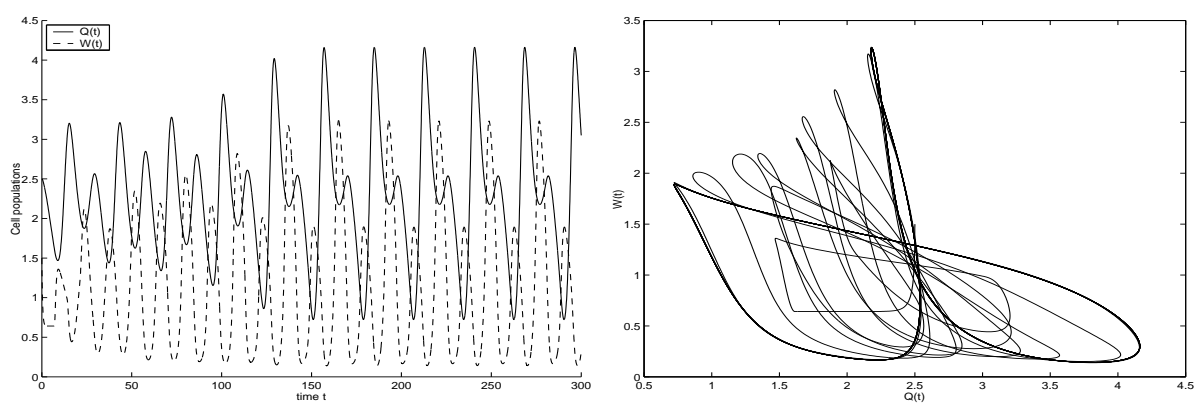

(a)
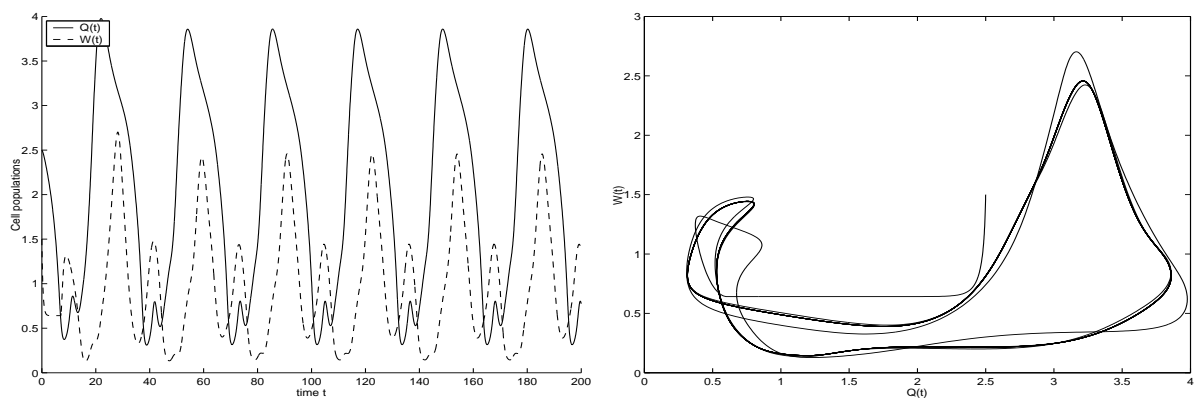

(b)
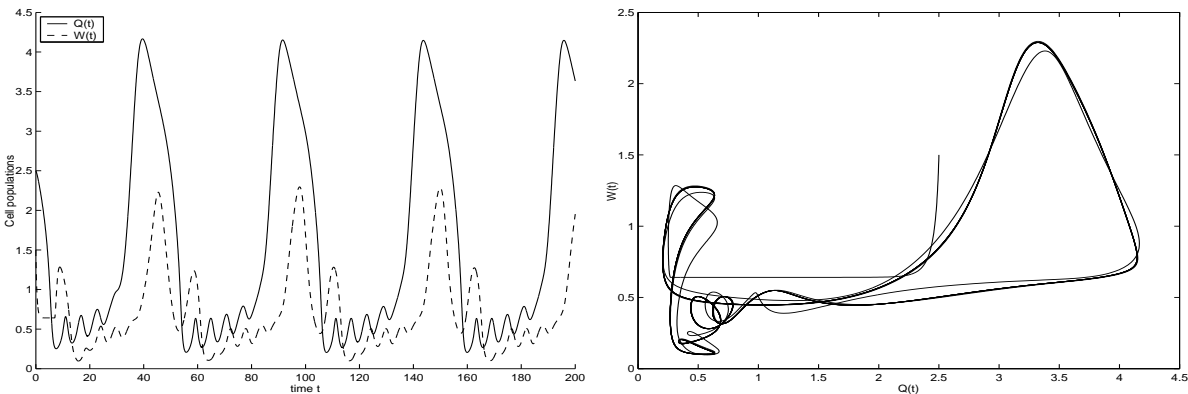

(c)

Figure 5: Long period oscillations. The solid line represents the hematopoietic stem cells population and the dashed line the white blood cell population. Parameters are given by (18) and (19), with $A=20$. The time delays are (a) $\tau_{1}=3$ days, (b) $\tau_{1}=4$ days, (c) $\tau_{1}=5$ days, with $\tau_{2}=7$ days in the three cases. Oscillating solutions with periods in the range of (a) 20-25 days, (b) 30 days and (c) 50 days, for the two cell populations, are observed, that can be related to oscillations observed in cyclical neutropenia (see [9]). Ripples can be observed in the two populations in figure (c). 


\section{Appendix}

\section{A Existence of a Unique Positive Equilibrium: Proof of Proposition 2.1}

We assume that condition (5) holds true and that the function $Q \mapsto Q \beta(Q)$ is decreasing on the interval $\left(Q_{0}, Q_{1}\right)$, where $Q_{0}$ and $Q_{1}$ are defined in (6).

In the following, for the sake of simplicity, we set

$$
\xi=2 e^{-\gamma_{1} \tau_{1}}-1
$$

From (6), $Q_{0}>0$ is the unique solution of

$$
\xi \beta\left(Q_{0}\right)=k(0)+K
$$

and $Q_{1}>Q_{0}$ is the unique solution of

$$
\xi \beta\left(Q_{1}\right)=K \text {. }
$$

The existence and uniqueness of $Q_{0}$ and $Q_{1}$ follow from the fact that $\beta$ is decreasing, tends to zero at infinity and satisfies (5).

Since $k$ is a positive decreasing function, we define $k^{-1}:(0, k(0)] \rightarrow[0,+\infty)$ as its inverse function. Then system (3)-(4) is equivalent to

$$
W^{*}=k^{-1}\left(\xi \beta\left(Q^{*}\right)-K\right)
$$

and

$$
\xi A Q^{*} \beta\left(Q^{*}\right)-\gamma_{2} k^{-1}\left(\xi \beta\left(Q^{*}\right)-K\right)=A K Q^{*}
$$

with $Q^{*} \in\left(Q_{0}, Q_{1}\right)$.

For $Q \in\left[Q_{0}, Q_{1}\right)$, set

$$
\chi(Q)=\xi A Q \beta(Q)-\gamma_{2} k^{-1}(\xi \beta(Q)-K) .
$$

Note that for $Q \in\left[Q_{0}, Q_{1}\right)$, we have $\xi \beta(Q)-K \in(0, k(0)]$, so $\chi$ is well-defined.

We first check that, from (20),

$$
\chi\left(Q_{0}\right)=A(k(0)+K) Q_{0}-\gamma_{2} k^{-1}(k(0))=A(k(0)+K) Q_{0}>A K Q_{0} .
$$

Moreover, from (21),

$$
\lim _{Q \rightarrow Q_{1}} k^{-1}(\xi \beta(Q)-K)=+\infty .
$$

Thus,

$$
\lim _{Q \rightarrow Q_{1}} \chi(Q)=-\infty
$$

Therefore, since the mapping $Q \mapsto A K Q$ is increasing on $\left[Q_{0}, Q_{1}\right), \chi\left(Q_{0}\right)>A K Q_{0}$ and $\lim _{Q \rightarrow Q_{1}} \chi(Q)=-\infty$, there exists at least one $Q^{*}>0$ such that

$$
\chi\left(Q^{*}\right)=A K Q^{*} \quad \text { with } Q^{*} \in\left(Q_{0}, Q_{1}\right) .
$$

Furthermore, on the interval $\left[Q_{0}, Q_{1}\right)$, the function $Q \mapsto \xi \beta(Q)-K$ is decreasing. Since $k^{-1}$ is decreasing, the mapping $Q \mapsto k^{-1}(\xi \beta(Q)-K)$ is increasing on $\left[Q_{0}, Q_{1}\right)$, and $Q \mapsto$ $-\gamma_{2} k^{-1}(\xi \beta(Q)-K)$ is decreasing. Since we assume that $Q \mapsto Q \beta(Q)$ is decreasing on the interval $\left(Q_{0}, Q_{1}\right)$, it follows that $\chi$ is decreasing. Consequently $Q^{*}$ is unique. 


\section{B Properties of Purely Imaginary Roots: Proof of Lemma}

\section{1}

From the arguments in section 3, before Lemma 3.1, when $\tau_{2}=\tau_{2, l}^{*}$, then $\pm i \omega_{l}$ is a pair of pure imaginary roots of (12).

Consider the branch of characteristic roots $\lambda\left(\tau_{2}\right)=\nu\left(\tau_{2}\right)+i \omega\left(\tau_{2}\right)$ of $(12)$, with

$$
\nu\left(\tau_{2, l}^{*}\right)=0 \quad \text { and } \quad \omega\left(\tau_{2, l}^{*}\right)=\omega_{l} .
$$

By differentiating (12) with respect to $\tau_{2}$, we obtain

$$
\left[2 \lambda+b_{1}+\left(b_{3}-\tau_{2}\left(b_{3} \lambda+b_{4}\right)\right) e^{-\lambda \tau_{2}}\right] \frac{d \lambda}{d \tau_{2}}=\lambda\left(b_{3} \lambda+b_{4}\right) e^{-\lambda \tau_{2}} .
$$

If we suppose, by contradiction, that $\lambda\left(\tau_{2, l}^{*}\right)$ is not a simple root of $(12)$, then we obtain

$$
i \omega_{l}\left(i b_{3} \omega_{l}+b_{4}\right) e^{-i \omega_{l} \tau_{2, l}^{*}}=0 .
$$

Separating real and imaginary parts in the above equality, we deduce

$$
\begin{aligned}
-b_{3} \omega_{l}^{2} \cos \left(\omega_{l} \tau_{2, l}^{*}\right)+b_{4} \omega_{l} \sin \left(\omega_{l} \tau_{2, l}^{*}\right) & =0, \\
b_{4} \omega_{l} \cos \left(\omega_{l} \tau_{2, l}^{*}\right)+b_{3} \omega_{l}^{2} \sin \left(\omega_{l} \tau_{2, l}^{*}\right) & =0 .
\end{aligned}
$$

Since $i \omega_{l}$ is a root of (12) when $\tau_{2}=\tau_{2, l}^{*}$, we deduce by separating real and imaginary parts in (12) that

$$
\begin{aligned}
-b_{3} \omega_{l} \cos \left(\omega_{l} \tau_{2, l}^{*}\right)+b_{4} \sin \left(\omega_{l} \tau_{2, l}^{*}\right) & =b_{1} \omega_{l}, \\
b_{4} \cos \left(\omega_{l} \tau_{2, l}^{*}\right)+b_{3} \omega_{l} \sin \left(\omega_{l} \tau_{2, l}^{*}\right) & =\omega_{l}^{2}-b_{2} .
\end{aligned}
$$

Consequently, using (24) in (23) and the fact that $\omega_{l}>0$, we deduce that

$$
b_{1}=0 \quad \text { and } \quad \omega_{l}^{2}=b_{2} .
$$

Since $b_{1}>0$, we obtain a contradiction. We conclude that $\pm i \omega_{l}$ are simple roots of (12).

Now, using (22) we deduce that

$$
\begin{aligned}
\left(\frac{d \lambda}{d \tau_{2}}\right)^{-1} & =\frac{2 \lambda+b_{1}+\left[b_{3}-\tau_{2}\left(b_{3} \lambda+b_{4}\right)\right] e^{-\lambda \tau_{2}}}{\lambda\left(b_{3} \lambda+b_{4}\right) e^{-\lambda \tau_{2}}} \\
& =\frac{\left(2 \lambda+b_{1}\right) e^{\lambda \tau_{2}}+b_{3}}{\lambda\left(b_{3} \lambda+b_{4}\right)}-\frac{\tau_{2}}{\lambda}
\end{aligned}
$$

From (12),

$$
e^{\lambda \tau_{2}}=-\frac{b_{3} \lambda+b_{4}}{\lambda^{2}+b_{1} \lambda+b_{2}}
$$

so we obtain

$$
\left(\frac{d \lambda}{d \tau_{2}}\right)^{-1}=-\frac{\left(2 \lambda+b_{1}\right)}{\lambda\left(\lambda^{2}+b_{1} \lambda+b_{2}\right)}+\frac{b_{3}}{\lambda\left(b_{3} \lambda+b_{4}\right)}-\frac{\tau_{2}}{\lambda} .
$$

Then,

$$
\begin{aligned}
\left(\frac{d \lambda}{d \tau_{2}}\right)_{\tau_{2}=\tau_{2, l}^{*}}^{-1} & =\frac{-2 i \omega_{l}-b_{1}}{i \omega_{l}\left(-\omega_{l}^{2}+b_{2}+i b_{1} \omega_{l}\right)}+\frac{b_{3}}{i \omega_{l}\left(i b_{3} \omega_{l}+b_{4}\right)}-\frac{\tau_{2, l}^{*}}{i \omega_{l}}, \\
& =\frac{-2 i \omega_{l}-b_{1}}{i\left(b_{2}-\omega_{l}^{2}\right) \omega_{l}-b_{1} \omega_{l}^{2}}+\frac{b_{3}}{-b_{3} \omega_{l}^{2}+i b_{4} \omega_{l}}-\frac{\tau_{2, l}^{*}}{i \omega_{l}} .
\end{aligned}
$$


Consequently,

$$
\begin{aligned}
\operatorname{Re}\left(\frac{d \lambda}{d \tau_{2}}\right)_{\tau_{2}=\tau_{2, l}^{*}}^{-1} & =\frac{b_{1}^{2} \omega_{l}^{2}-2 \omega_{l}^{2}\left(b_{2}-\omega_{l}^{2}\right)}{\left(b_{2}-\omega_{l}^{2}\right)^{2} \omega_{l}^{2}+b_{1}^{2} \omega_{l}^{4}}-\frac{b_{3}^{2} \omega_{l}^{2}}{b_{3}^{2} \omega_{l}^{4}+b_{4}^{2} \omega_{l}^{2}}, \\
& =\frac{b_{1}^{2}-2 b_{2}+2 \omega_{l}^{2}}{\left(b_{2}-\omega_{l}^{2}\right)^{2}+b_{1}^{2} \omega_{l}^{2}}-\frac{b_{3}^{2}}{b_{3}^{2} \omega_{l}^{2}+b_{4}^{2}} .
\end{aligned}
$$

Since $\omega_{l}^{2}$ is a root of $F$, it follows from (16) that

$$
b_{3}^{2} \omega_{l}^{2}+b_{4}^{2}=\omega_{l}^{4}+\left(b_{1}^{2}-2 b_{2}\right) \omega_{l}^{2}+b_{2}^{2}=\left(b_{2}-\omega_{l}^{2}\right)^{2}+b_{1}^{2} \omega_{l}^{2} .
$$

This yields

$$
\operatorname{Re}\left(\frac{d \lambda}{d \tau_{2}}\right)_{\tau_{2}=\tau_{2, l}^{*}}^{-1}=\frac{b_{1}^{2}-2 b_{2}-b_{3}^{2}+2 \omega_{l}^{2}}{\left(b_{2}-\omega_{l}^{2}\right)^{2}+b_{1}^{2} \omega_{l}^{2}}=\frac{F^{\prime}\left(\omega_{l}^{2}\right)}{\left(b_{2}-\omega_{l}^{2}\right)^{2}+b_{1}^{2} \omega_{l}^{2}}
$$

Since

$$
\operatorname{sign}\left\{\operatorname{Re}\left(\frac{d \lambda}{d \tau_{2}}\right)_{\tau_{2}=\tau_{2, l}^{*}}^{-1}\right\}=\operatorname{sign}\left\{\left.\frac{d \operatorname{Re}(\lambda)}{d \tau_{2}}\right|_{\tau=\tau_{2, l}^{*}}\right\},
$$

we obtain

$$
\operatorname{sign}\left\{\left.\frac{d \operatorname{Re}(\lambda)}{d \tau_{2}}\right|_{\tau=\tau_{2, l}^{*}}\right\}=\operatorname{sign}\left\{F^{\prime}\left(\omega_{l}^{2}\right)\right\} .
$$

\section{References}

[1] Adimy, M., Crauste, F., 2003. Global stability of a partial differential equation with distributed delay due to cellular replication. Nonlinear Analysis 54 (8), 1469-1491, doi:10.1016/S0362-546X(03)00197-4.

[2] Adimy, M., Crauste, F., 2005. Existence, positivity and stability for a nonlinear model of cellular proliferation. Nonlinear Analysis: Real World Applications 6 (2), 337-366, doi:10.1016/j.nonrwa.2004.09.001.

[3] Adimy, M., Crauste, F., Halanay, A., Neamţu, M., Opriş, D, 2006. Stability of limit cycles in a pluripotent stem cell dynamics model. Chaos, Solitons and Fractals 27 (4), 1091-1107, doi:10.1016/j.chaos.2005.04.083.

[4] Adimy, M., Crauste, F., Pujo-Menjouet, L., 2005a. On the stability of a maturity structured model of cellular proliferation. Discret. Cont. Dyn. Sys. Ser. A 12 (3), 501-522.

[5] Adimy, M., Crauste, F., Ruan, S., 2005b. A mathematical study of the hematopoiesis process with applications to chronic myelogenous leukemia. SIAM J. Appl. Math. 65 (4), 1328-1352.

[6] Adimy, M., Crauste, F., Ruan, S., 2005c. Stability and Hopf bifurcation in a mathematical model of pluripotent stem cell dynamics. Nonlinear Analysis: Real World Applications 6 (4), 651-670, doi:10.1016/j.nonrwa.2004.12.010.

[7] Adimy, M., Pujo-Menjouet, L., 2003. Asymptotic behaviour of a singular transport equation modelling cell division. Discret. Cont. Dyn. Sys. Ser. B 3, 439-456. 
[8] Beretta, E., Kuang, Y., 2002. Geometric stability switch criteria in delay differential systems with delay dependent parameters. SIAM J. Math. Anal. 33, 1144-1165.

[9] Bernard, S., Bélair, J., Mackey, M. C., 2003. Oscillations in cyclical neutropenia: New evidence based on mathematical modeling. J. Theor. Biol. 223, 283-298, doi:10.1016/S0022-5193(03)00090-0.

[10] Bernard, S., Bélair, J., Mackey, M. C., 2004. Bifurcations in a white-blood-cell production model. C. R. Biologies 327, 201-210, doi:10.1016/j.crvi.2003.05.005.

[11] Boese, F. G., 1995. Stability criteria for second-order dynamical systems involving several time delays. SIAM J. Math. Anal. 26, 1306-1330.

[12] Burns, F. J., Tannock, I. F., 1970. On the existence of a G $_{0}$ phase in the cell cycle. Cell Tissue Kinet. 19, 321-334.

[13] Colijn, C., Mackey, M. C., 2005a. A mathematical model of hematopoiesis - I. Periodic chronic myelogenous leukemia. J. Theor. Biol. 237, 117-132, doi:10.1016/j.jtbi.2005.03.033.

[14] Colijn, C., Mackey, M. C., 2005b. A mathematical model of hematopoiesis - II. Cyclical neutropenia. J. Theor. Biol. 237, 133-146, doi:10.1016/j.jtbi.2005.03.034.

[15] Dyson, J., Villella-Bressan, R., Webb, G. F., 1996. A singular transport equation modelling a proliferating maturity structured cell population. Can. Appl. Math. Quart. 4, $65-95$.

[16] Dyson, J., Villella-Bressan, R., Webb, G. F., 1998. An age and maturity structured model of cell population dynamics, in: Horn, M., Simonnett, G., Webb, G. F. (Eds), Mathematical Models in Medical and Health Science, Vanderbilt University Press, Nashville, TN, pp. 66-116.

[17] Dyson, J., Villella-Bressan, R., Webb, G. F., 2000a. A nonlinear age and maturity structured model of population dynamics, I: Basic theory. J. Math. Anal. Appl. 242 (1), 93-104, doi:10.1006/jmaa.1999.6656.

[18] Dyson, J., Villella-Bressan, R., Webb, G. F., 2000b. A nonlinear age and maturity structured model of population dynamics, II: Chaos. J. Math. Anal. Appl. 242 (2), 255-270, doi:10.1006/jmaa.1999.6657.

[19] Fortin, P., Mackey, M. C., 1999. Periodic chronic myelogenous leukemia: Spectral analysis of blood cell counts and etiological implications. Brit. J. Haematol. 104, 336-345.

[20] Hale, J., Verduyn Lunel, S. M., 1993. Introduction to Functional Differential Equations, Applied Mathematical Sciences Vol. 99, New York: Springer-Verlag.

[21] Haurie, C., Dale, D. C., Mackey, M. C., 1998. Cyclical neutropenia and other periodic hematological diseases: A review of mechanisms and mathematical models. Blood 92, 2629-2640.

[22] Haurie, C., Dale, D. C., Mackey, M. C., 1999. Occurrence of periodic oscillations in the differential blood counts of congenital, idiopathic and cyclical neutropenic patients before and during treatment with G-CSF. Exper. Hematol. 27, 401-409, doi:10.1016/S0301472X(98)00061-7. 
[23] Hearn, T., Haurie, C., Mackey, M. C., 1998. Cyclical neutropenia and the peripheral control of white blood cell production. J. Theor. Biol. 192, 167-181, doi:10.1006/jtbi.1997.0589.

[24] Lajtha, L. G., 1959. On DNA labeling in the study of the dynamics of bone marrow cell populations, in: Stohlman, Jr., F. (Ed), The Kinetics of Cellular Proliferation, Grune and Stratton, New York, pp. 173-182.

[25] MacDonald, N., 1978. Cyclical neutropenia: Models with two cell types and two time lags, in: Valleron, A, Macdonald, P (Eds), Biomathematics and Cell Kinetics, NorthHolland, Amsterdam, pp. 287-295.

[26] Mackey, M. C., 1978. Unified hypothesis of the origin of aplastic anaemia and periodic hematopoïesis, Blood 51, 941-956.

[27] Mackey, M. C., 2001. Cell kinetic status of haematopoietic stem cells, Cell Prolif. 34, 71-83.

[28] Mackey, M. C., Rey, A., 1993. Multistability and boundary layer development in a transport equation with retarded arguments. Can. Appl. Math. Quart. 1, 1-21.

[29] Mackey, M. C., Rey, A., 1995a. Propagation of population pulses and fronts in a cell replication problem: non-locality and dependence on the initial function. Physica D 86, 373-395, doi:10.1016/0167-2789(95)00114-J.

[30] Mackey, M. C., Rey, A., 1995b. Transitions and kinematics of reaction-convection fronts in a cell population model. Physica D 80, 120-139, doi:10.1016/0167-2789(95)90067-5.

[31] Mackey, M. C., Rudnicki, R., 1994. Global stability in a delayed partial differential equation describing cellular replication, J. Math. Biol. 33, 89-109.

[32] Mackey, M. C., Rudnicki, R., 1999. A new criterion for the global stability of simultaneous cell replication and maturation processes, J. Math. Biol. 38, 195-219.

[33] Michor, F., Hughes, T. P., Iwasa, Y., Branford, S., Shah, N. P., Sawyers, C. L., Nowak, M. A., 2005. Dynamics of chronic myeloid leukemia, Nature 435, 1267-1270.

[34] Novak, J. P., Nečas, E., 1994. Proliferation differentiation pathways of murine haematopoiesis: correlation of lineage fluxes, Cell Prolif. 27, 597-633.

[35] Pujo-Menjouet, L., Bernard, S., Mackey, M. C., 2005. Long period oscillations in a $G_{0}$ model of hematopoietic stem cells. SIAM J. Appl. Dynam. Syst. 4 (2), 312-332.

[36] Pujo-Menjouet, L., Mackey, M. C., 2004. Contribution to the study of periodic chronic myelogenous leukemia. C. R. Biologies 327, 235-244, doi:10.1016/j.crvi.2003.05.004.

[37] Pujo-Menjouet, L., Rudnicki, R., 2000. Global stability of cellular populations with unequal division. Can. Appl. Math. Quart. 8 (2), 185-202.

[38] Ruan, S., Wei, J., 1999. Periodic solutions of planar systems with two delays. Proc. Royal Soc. Edinburgh Ser. 129A, 1017-1032.

[39] Ruan, S., Wei, J., 2003. On the zeros of transcendental functions with applications to stability of delay differential equations with two delays. Dyn. Contin. Discrete Impuls. Syst. Ser. A Math. Anal. 10, 863-874. 
[40] Shampine, L. F., Thompson, S., 2001. Solving DDEs in Matlab. Appl. Numer. Math. 37, 441-458, doi:10.1016/S0168-9274(00)00055-6. http://www.radford.edu/ thompson/webddes/.

[41] Wei, J., Ruan, S., 1999. Stability and bifurcation in a neural network model with two delays. Physica D 130, 255-272, doi:10.1016/S0167-2789(99)00009-3. 